\title{
Caesar Automatic Layout of UML Class Diagrams
}

\author{
Carsten Gutwenger ${ }^{1}$, Michael Jünger ${ }^{2}$, Karsten Klein ${ }^{1}$, Joachim Kupke ${ }^{1}$, \\ Sebastian Leipert ${ }^{1}$, and Petra Mutzel $^{3}$ \\ 1 Research Center caesar, Friedensplatz 16, D-53111 Bonn, Germany \\ \{gutwenger|kklein|kupke|leipert\}@caesar.de \\ 2 Universität zu Köln, Pohligstr. 1, D-50969 Köln, Germany \\ mjuenger@informatik. uni-koeln.de \\ 3 Technische Universität Wien, Favoritenstr. 9-11 E186, A-1040 Wien, Austria \\ mutzel@ads.tuwien.ac.at
}

\section{Short Description}

UML diagrams have become increasingly important in the engineering and reengineering processes for software systems. Of particular interest are UML class diagrams whose purpose is to display class hierarchies (generalizations), associations, aggregations, and compositions in one picture. The combination of hierarchical and non-hierarchical relations poses a special challenge to a graph layout tool. Commercial software typically uses Sugiyama-style methods, see, e.g., [1] that cannot properly distinguish between hierarchical and non-hierarchical relations.

The caesar graph drawing group develops and implements a library of algorithms and data structures for graph drawing. Special emphasis is on the layout of UML class diagrams.

\section{Areas of Application}

The software addresses users of UML-class diagrams such as software engineers and data base engineers.

\section{Layout Algorithms and Layout Features}

Our UML-class diagram layout tool applies state-of-the-art techniques for planarization, planar drawing, and compaction. In contrast to existing tools, the hierarchical and non-hierarchical relations are neither treated alike nor as separate tasks in a two-phase process as in, e.g., Seemann 2].

For a clear visualization of the specific combination of hierarchical and nonhierarchical components in UML Class Diagrams we put special emphasis on meeting a balanced mixture of the following aesthetic criteria: Crossing minimization, bend minimization, uniform direction within each hierarchical component, no nesting of one hierarchy within another, orthogonal layout, merging of multiple inheritance edges, good edge labeling.

P. Mutzel, M. Jünger, and S. Leipert (Eds.): GD 2001, LNCS 2265, pp. 461-462, 2002.

(C) Springer-Verlag Berlin Heidelberg 2002 


\section{Architecture}

The caesar $\mathrm{C}++$ library for graph drawing is an object oriented system using its own implementations of efficient data structures and algorithms.

The library runs on Microsoft and Unix systems. The Microsoft Visio Add-On operates on Windows NT/2000/98/Me/XP using Visio 2000 or Visio 2002.

\section{Interfaces}

The caesar library provides a generic layout interface that is independent of the drawing environment. The library currently provides an interface to Visio 2000/2002, a diagramming software by Microsoft.

\section{Screenshot}

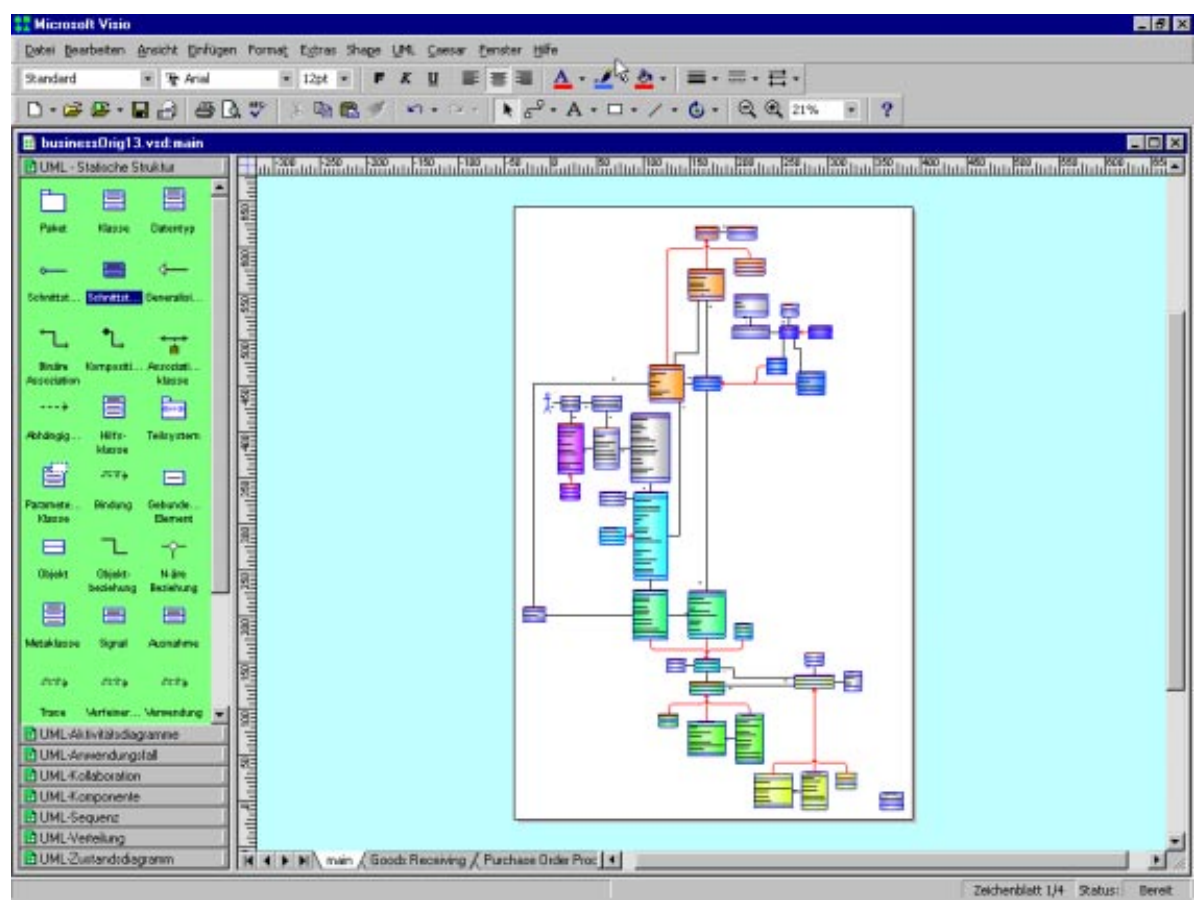

\section{References}

1. Rational Rose, 2001. Rational Software Corporation.

2. J. Seemann. Extending the Sugiyama algorithm for drawing UML class diagrams. In G. Di Battista, editor, Proc. Graph Drawing 1997 (GD '97), volume 1353 of Lecture Notes in Computer Science, pages 415-424. Springer, 1998. 